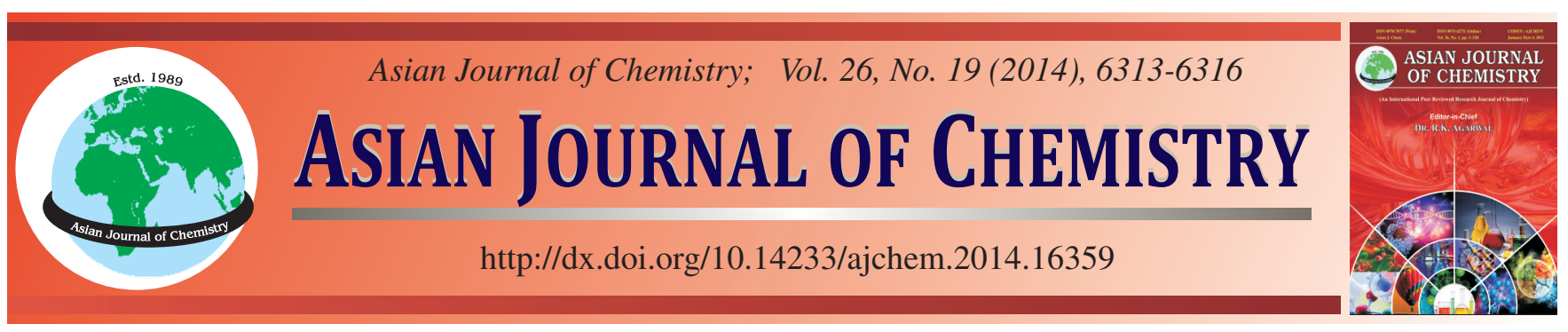

\title{
Modified Polyethylene for Synthetic Wood Applications
}

\author{
M.Y. AbdelaAL ${ }^{1, * \dagger}$, E.H. Elmossalamy ${ }^{1,2}$ and S.O.S. BAHAFFI ${ }^{1}$
}

${ }^{1}$ Chemistry Department, Faculty of Science, King Abdulaziz University, PO Box 80203, Jeddah 21589, Saudi Arabia

${ }^{2}$ Chemistry Department, Faculty of Science, Benha University, Benha, Egypt

${ }^{\dagger}$ Chemistry Department, Faculty of Science, Mansoura University, Mansoura 35516, Egypt

*Corresponding author: Fax: +966 2 6952293; Tel: +966 500096707; E-mail: myabdelaal@ gmail.com; magdyabdelaal@yahoo.com

\begin{abstract}
Polyethylene as a polyolefin has been grafted with maleic anhydride under optimized conditions to attain maleic anhydride grafted polyethylene with higher grafting yield. Maleic anhydride grafted polyethylene has been characterized with FT-IR spectroscopy and thermal analysis as well as some chemical tests. A suitable amount of saw dust as an example for natural fibers has been collected and prepared for hot press casting with polyethylene to attain wood plastic composite. Maleic anhydride grafted polyethylene was used as a coupling agent to enhance the compatibility between polyethylene and saw dust. Samples of wood plastic composite were prepared without maleic anhydride grafted polyethylene as blank. Wood plastic composite samples have been tested regarding mechanical, physical and chemical characteristics.
\end{abstract}

Keywords: Modification, Natural Fiber, Grafting, Polyolefins, Wood, Tensile Strength.

\section{INTRODUCTION}

Modification of polymers is a well known strategy to change the characteristics of the polymer according to the modification technique and extent to be suitable for the application field ${ }^{1-6}$. Composites are materials consisting of two or more identifiable constituents but not mixed to an atomic scale ${ }^{7}$ and are controlled by the properties of their individual compositions and interaction. The major constituents of wood-plastic composites are both the filler which is either wood or generally natural fibers and the matrix which can be thermoset or thermoplastic resins in addition to other chemical additives. The use of fillers in thermoplastic industry is a common way to increase stiffness and strength to plastics. Inorganic fillers are of the greatest in demand especially calcium carbonate, glass fiber, clay, talc and mica. The supposed environmentally friendly nature of organic fillers has attracted the industry and has led to a major shift in choosing filler types. The growth of wood plastic composites has shown a jump during the last two decades in the plastic industry and the acceptance of wood plastic composites which are currently used in various applications such as decking, roof tiles, siding, window and door frames attracted the attention of industry to the organic fillers ${ }^{8}$.

Numerous studies over the past decades on wood fiber plastic composites indicated that composites can be readily formed using compounding and compression molding technologies ${ }^{9}$. In most of those technologies, wood fiber either mixed with polymer or added as filler in it followed by pressing or molding under suitable conditions of pressure and temperature. Although different compounding techniques can be applied to produce wood plastic composites, twin screw extruder is still the standard in the industry resulting in production of high modulus composite with low rate of water absorption. However, there is no much investigation to know the effect of extrusion variables on characteristics of wood plastic composites $^{10-13}$. wood plastic composite covers several composites using polyolefins such as polypropylene, poly(vinyl chloride) and polyethylene and binders or fillers such as wood flour, flax fibers and other natural fibers ${ }^{14}$. Wood is a lignocellulosic material composed mainly of cellulose (50-65\%), reasonable content of lignin (20-25\%) in addition to some extractives and traces of ash (1-10\%). The ratio of constituents differs based on species of wood ${ }^{15}$.

At the beginning, wood plastic composite was formed by using recycled wood fibers or chips. Unfortunately, such composites products were ideal for relatively less demanding applications but there are increased efforts to produce wood plastic composites as a viable alternate use for residual plant fiber material and thermoplastics ${ }^{16-22}$. The new generations of wood plastic composites are high-end technology products which exhibit superior mechanical properties, high dimensional stability and can be extruded to high dimensional tolerances $^{23}$. This helps to form tough and stable complex shapes needed for engineering applications ${ }^{8}$. 
The advantages of wood plastic composites are their processing ability; wide range and inexpensive raw materials which can be wastes of wood and recycled plastics and their competitive price with conventional supplies such as medium density fiberboard and PVC in addition to their availability in several finishes and appearances. Hence, wastes of wood and recycled plastics become useful resources by converting them into wood plastic composite instead of causing environmental concerns. Despite the advantages of wood plastic composite over wood and plastic, the interaction between wood and plastic at fiber-matrix interface does not necessarily yield strong bonds ${ }^{24}$. The major reason for weak bonds has been attributed to the polar nature of the wood (hydroxyl groups) while some plastics such as polyethylene and polypropylene are non-polar polymers ${ }^{25}$. It is necessary to modify the wood fiber with a proper coupling agents or coating the fiber with appropriate surface modifying resin to develop composites with superior bonds at the boundary which is a significant factor to obtain composites of improved mechanical proper$\operatorname{ties}^{26}$. The other alternative is to modify the plastic component in the wood plastic composite either by using blends with different plastic types or by using coupling agent which may be compatible with the hydrophobic plastic components and the hydrophilic wood or natural fiber component. In such cases grafted polypropylene and/or polyethylene with maleic anhydride can be of benefits in this regard ${ }^{27}$.

\section{EXPERIMENTAL}

All chemicals are purchased by Aldrich unless otherwise mentioned. Thermal and FT-IR Spectroscopic analyses used for characterization of maleic anhydride grafted polyethylene were achieved in the department. Tensile testing has been also performed in the Faculty of Engineering, King Abdulaziz University at Saudi Arabia.

Preparation of maleic anhydride grafted polyethylene: A mixture of $70 \mathrm{~g}$ of polyethylene (2.5 mole), $49 \mathrm{~g}$ of maleic anhydride ( 0.5 mole) and $1.2 \mathrm{~g}$ of benzoylperoxide ( 5 mmole) was added to $250 \mathrm{~mL}$ of benzene. The mixture was kept under reflux with stirring for $2 \mathrm{~h}$ and then left to cool while stirring until the temperature reduced to $60{ }^{\circ} \mathrm{C}$. The reaction mixture has been poured onto a large amount of ethanol while stirring. The obtained product has been collected by filtration, air dried at $50{ }^{\circ} \mathrm{C}$ under reduced pressure and prepared for characterization by using FT-IR spectroscopic analysis.

Sample preparation: Saw dust used as reinforcing material was a mixture of different wood species collected from public carpentries in Jeddah, Saudi Arabia with particle size ranged between 80-100 mesh. Commercial polyethylene produced by the Saudi Basic Industrial Corp. (SABIC), Saudi Arabia was converted into small particles. A maleated polyethylene (MAPE) was prepared by grafting of polyethylene with maleic anhydride as mentioned above and used as a coupling agent.

Compounding: Saw dust was dried at $40{ }^{\circ} \mathrm{C}$ under reduced pressure for $24 \mathrm{~h}$ to moisture content of about $2 \%$ and then kept in sealed bags until required. The different samples were prepared as pellets through melt blending, extrusion and palletizing at $120{ }^{\circ} \mathrm{C}$ using a laboratory size twin-screw extruder.
The test specimens were obtained by melt press molding of the obtained pellets after drying at $80^{\circ} \mathrm{C}$ for $24 \mathrm{~h}$. All samples were melt press molded using a melt press of $10 \mathrm{kN} / \mathrm{cm}^{2}$. Table1 shows the composition of samples with saw dust content up to $45 \%$ based on the total weight of the mixture prior to compounding and in presence of $5 \%$ maleic anhydride grafted polyethylene as a coupling agent.

TABLE-1
\begin{tabular}{lccccc} 
COMPOSITION (\%) OF THE POLYETHYLENE-SAW \\
DUST BLENDS \\
\hline \multicolumn{1}{c}{ Component } & 100 & 95 & 85 & 75 & 55 \\
\hline Polyethylene & 0 & 5 & 15 & 25 & 45 \\
Saw dust & 5 & 5 & 5 & 5 & 5 \\
Maleic anhydride grafted polyethylene &
\end{tabular}

FT-IR spectroscopic analysis: Samples of polyethylene, maleic anhydride and maleic anhydride grafted polyethylene have been characterized by using FT-IR Spectroscopic analysis. The spectra of the above mentioned samples are shown in Fig. 1.

Thermal analysis: Samples of polyethylene, saw dust and wood plastic composite have been characterized by using thermo-gravimetric analysis (DTG) in air and with heating rate of $10^{\circ} \mathrm{C} / \mathrm{min}$. Results are shown in Figs. 2-4.

Tensile strength measurements: Tensile strength was measured for polyethylene-saw dust wood plastic composite samples with a Universal Testing Machine at the Faculty of Engineering, KAU, Saudi Arabia. Test specimens were of $\mathrm{W}=$ $19 \mathrm{~mm}, \mathrm{~L}=115 \mathrm{~mm}$ and $\mathrm{T}=3.5 \mathrm{~mm}$ dimensions as shown in Fig. 5 and the tests were conducted at a speed of $100 \mathrm{~mm} / \mathrm{min}$. Fig. 6 shows the average values of 5 replicates for each condition to obtain more representative results.

\section{RESULTS AND DISCUSSION}

There are different ways for grafting of polyethylene with maleic anhydride including melt extrusion in presence of a suitable initiator. This way has some drawbacks in the light of the processing ability of the grafting mixture during extrusion. In the current work, maleic anhydride has been grafted onto polyethylene using benzene as a liquid phase to facilitate good distribution of maleic anhydride over the amount of polyethylene to be grafted. Moreover, conducting the grafting process in presence of benzene keeps the ambient temperature low and consequently the adverse effect of higher temperature during the extrusion will be minimized.

FT-IR spectroscopy of maleic anhydride grafted polyethylene: Grafting of maleic anhydride onto polyethylene chains was confirmed by FT-IR spectroscopy and the relative percentage of maleic anhydride grafting in maleic anhydride grafted polyethylene can be predicted. FTIR spectra were recorded from 5000 to $400 \mathrm{~cm}^{-1}$. The relative under-peak area of the anhydride absorption between 1860 and $1710 \mathrm{~cm}^{-1}$ to the $\mathrm{CH}$ stretching peak at $720 \mathrm{~cm}^{-1}$ was considered as an approximate indicator for the grafting extent of maleic anhydride.

Samples were prepared by hot press under $10 \mathrm{kN}$ force at $200{ }^{\circ} \mathrm{C}$ to form thin films. Fig. 1 shows the FTIR spectra of maleic anhydride grafted polyethylene in addition to those of polyethylene and maleic anhydride for comparison. It is 
obvious from this figure the presence of new peaks 1720 and $1787 \mathrm{~cm}^{-1}$ when maleic anhydride is grafted successfully onto polyethylene. The bands at 1787 and $1720 \mathrm{~cm}^{-1}$ are due to the asymmetric and symmetric stretching modes, respectively, of carbonyl $(\mathrm{C}=\mathrm{O})$ groups of saturated maleic anhydride residues grafted onto polyethylene ${ }^{28-30}$.

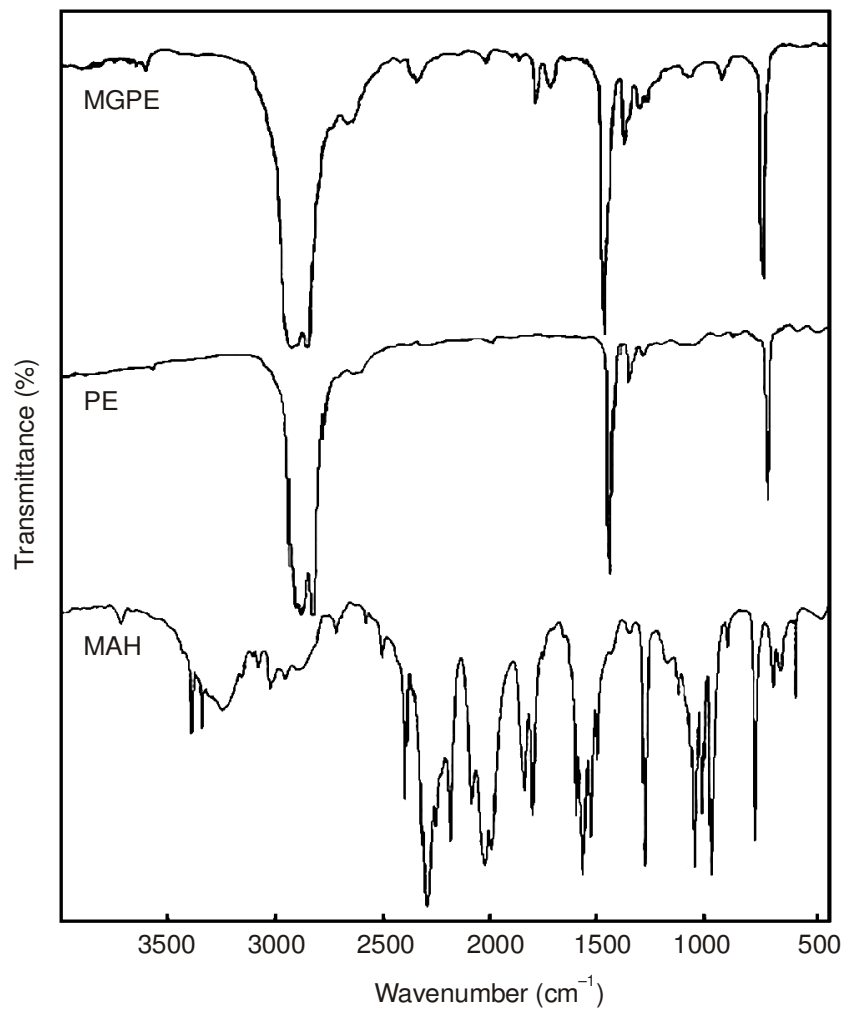

Fig. 1. FT-IR spectra of polyethylene, maleic anhydride and maleic anhydride grafted polyethylene

Thermal analysis: It is known that $\mathrm{T}_{\mathrm{g}}$ of polyethylene is about $20{ }^{\circ} \mathrm{C}$ and the melting temperature $\left(\mathrm{T}_{\mathrm{m}}\right)$ is $130{ }^{\circ} \mathrm{C}$. Also the ash ignition temperature is $340{ }^{\circ} \mathrm{C}$ while the self-ignition temperature in air is $350{ }^{\circ} \mathrm{C}$. In the current study, polyethylene sample was heated in air. Fig. 2 shows that the endothermic peaks occur around $138^{\circ} \mathrm{C}$. Fig. 3 shows the thermal analysis data of the saw dust in air where an endothermic peak occurs around $355{ }^{\circ} \mathrm{C}$ representing thermal degradation. There are also two exothermic peaks observed around $328^{\circ} \mathrm{C}$ and $445^{\circ} \mathrm{C}$ resulting from combustion.

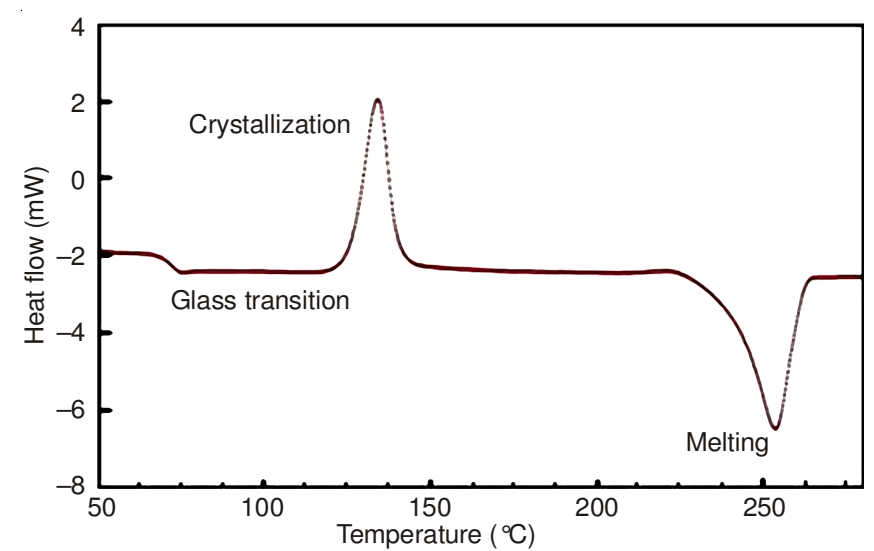

Fig. 2. Differential scanning calorimetric analysis of polyethylene

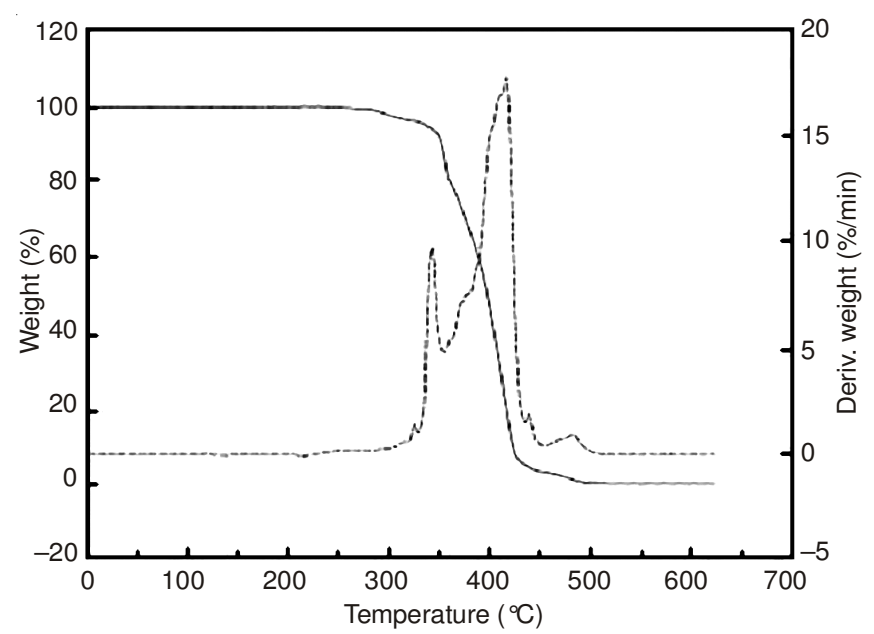

Fig. 3. TG-DTG plot of polyethylene in air at heating rate of $10{ }^{\circ} \mathrm{C} / \mathrm{min}$

Fig. 4 shows the TG-DTG plots of wood plastic composite composed of polyethylene and saw dust where the peak at $315^{\circ} \mathrm{C}$ results mainly from polyethylene while that at $355^{\circ} \mathrm{C}$ results from both polyethylene and saw dust. In addition, there is a peak at $435{ }^{\circ} \mathrm{C}$ which corresponds to polyethylene only. This means that the addition of saw dust facilitates the oxidation and combustion since wood is more combustible than polyethylene.

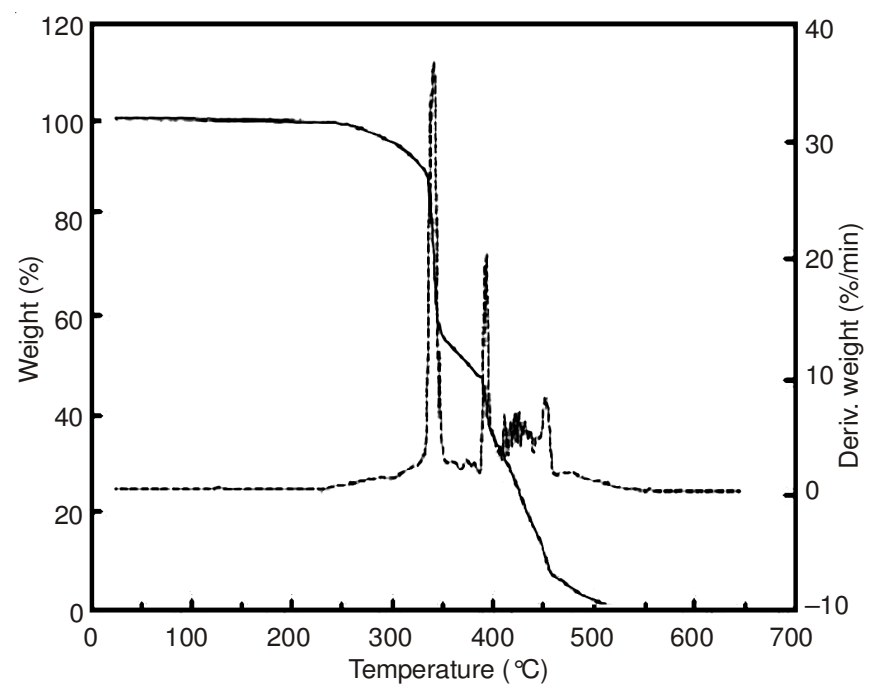

Fig. 4. TG-DTG of wood plastic composite sample in air at heating rate of $10^{\circ} \mathrm{C} / \mathrm{min}$

Tensile strength: Wood plastic composite samples used for tensile testing were of the dimensions $\mathrm{W}=19 \mathrm{~mm}, \mathrm{~L}=115$ $\mathrm{mm}$ and $\mathrm{T}=3.5 \mathrm{~mm}$ as shown in Fig. 5. Tests were conducted by using of a universal tensile testing machine at a speed of $100 \mathrm{~mm} / \mathrm{min}$ for 5 replicates.

Blank samples of wood plastic composite with no maleic anhydride grafted polyethylene showed tensile strength lower than those containing maleic anhydride grafted polyethylene as coupling agent. This behaviour was shown by all samples of different saw dust contents up to $45 \%$. The maximum tensile strength for the blank samples was $38.02 \mathrm{~N} / \mathrm{mm}^{2}$ while it was $52.06 \mathrm{~N} / \mathrm{mm}^{2}$ for the samples with maleic anhydride grafted polyethylene as coupling agent as shown in Fig. 6. This reflects 


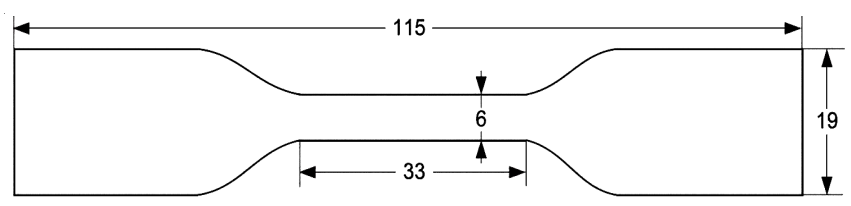

Fig. 5. Dimensions in mm of wood plastic composite test specimens for tensile strength test. Sample thickness for mechanical tests $=3.5 \mathrm{~mm}$

the great impact of maleic anhydride grafted polyethylene on the compatibility of wood plastic composite components.

Fig. 6 shows the dependence of tensile characteristics on the content (wt \%) of saw dust in presence of $5 \%$ of maleic anhydride grafted polyethylene as coupling agent and in absence of maleic anhydride grafted polyethylene as a blank. The tensile strength of the blank was $38.02 \mathrm{~N} / \mathrm{mm}^{2}$ at $0 \%$ saw dust content and decreased by increasing the saw dust content of the sample up to $45 \mathrm{wt} \%$ leading to decrease of the tensile strength to $34.32 \mathrm{~N} / \mathrm{mm}^{2}$. This means that the addition of saw dust up to $45 \mathrm{wt} \%$ leads to reduction in the tensile strength of $9.7 \%$ in comparison with pure polyethylene. This is attributable to the incompatibility of the hydrophobic polyethylene with saw dust which contains hydrophilic functionalities. This has been reflected as a decrease in the bonding strength between polyethylene and saw dust.

On the other hand, samples with maleic anhydride grafted polyethylene as coupling agent showed tensile strength of 39 $\mathrm{N} / \mathrm{mm}^{2}$ at $0 \%$ saw dust content and increased up to 52.06 $\mathrm{N} / \mathrm{mm}^{2}$ at $45 \mathrm{wt} \%$ of saw dust as shown in Fig. 6. It is obvious that the addition of maleic anhydride grafted polyethylene significantly increased the tensile strength almost in linear manner. This can be attributed to a stronger bonding between polyethylene and saw dust in presence of maleic anhydride grafted polyethylene as coupling agent which contains both hydrophobic and hydrophilic natures represented by polyethylene chains and maleic anhydride functionality added through grafting.

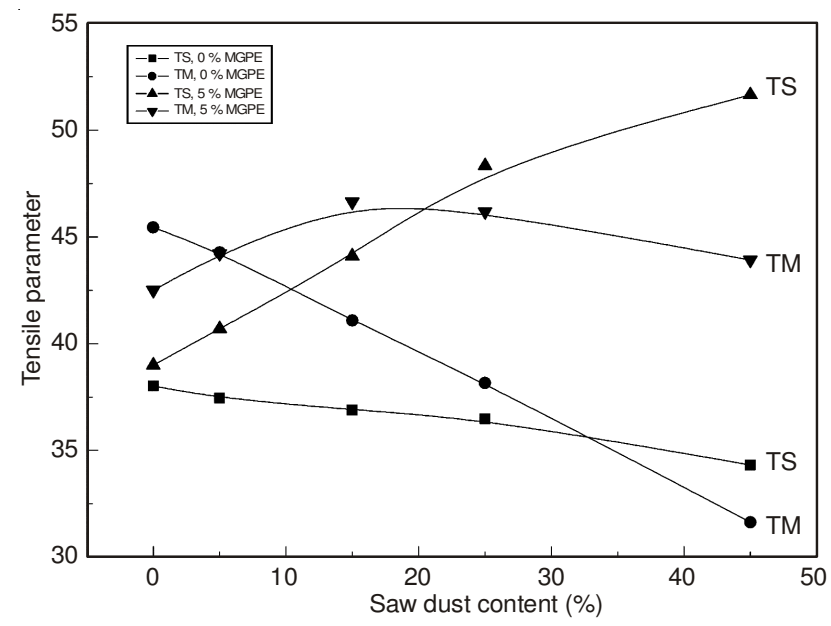

Fig. 6. Tensile strength and tensile modulus of wood plastic composite samples

\section{Conclusion}

From the current study, it can be concluded that the modification of polyethylene with maleic anhydride leads to promising materials derived from the different inexpensive polyolefins available in the market as waste material. The modified polyolefins serve as good candidates to attain better compatibility with natural fibers depending on the application directions. The tensile strength decreased with increasing saw dust due to the weak bonding with polyethylene. Utilization of maleic anhydride grafted polyethylene increased the tensile strength in comparison with pure polyethylene.

\section{ACKNOWLEDGEMENTS}

This project was funded by the Saudi Basic Industries Corporation (SABIC) and the Deanship of Scientific Research (DSR), King Abdulaziz University, Jeddah, under grant \# MS/ 12/276. The authors, therefore, acknowledged with thanks SABIC and DSR technical and financial support.

\section{REFERENCES}

1. M.Y. Abdelaal, T.R. Sobahi and H.F. Al-Shareef, Int. J. Biol. Macromol., 55, 231 (2013).

2. T.R. Sobahi, M.Y. Abdelaal and M.S.I. Makki, Arab. J. Chem. (In press).

3. M.Y.Abdelaal, T.R. Sobahi and M.S.I. Makki, Construct. Build. Mater., 25, 3267 (2011).

4. M.A. Salam, M.S.I. Makki and M.Y.A. Abdelaal, J. Alloys Comp., 509, 2582 (2011).

5. M.Y. Abdelaal and T.R. Sobahi, J. Appl. Polym. Sci., 104, 2304 (2007).

6. S.O.S. Bahaffi, M.Y. Abdelaal and E.A. Assirey, Int. J. Polym. Mater., 55, 477 (2006).

7. J.W. Weeton, D.M. Peters and K.L. Thomas, American Society for Metals Park, Engineers' Guide to Composites Materials, Ohio, U.S.A., pp. 1-1 to 8-41 (1987).

8. C. Eckert, Opportunities for Natural Fibers in Plastic Composites: In Proc. Progress in Wood Fiber-Plastic Composites Conference, May 25-26, Toronto, Canada (2000).

9. D.V. Maldas, Industrial Perspective of Cellulose in Thermoplastic Composites; In Handbook of Engineering and Polymeric Materials, pp. 577-584 (1997).

10. S.-K. Yeh and R.K. Gupta, Composites A, 39, 1694 (2008).

11. T. Fakhrul and M.A. Islam, Procedia Eng., 56, 795 (2013).

12. N. Ayrilmis, Composites B, 44, 745 (2013).

13. Z.N. Azwa, B.F. Yousif, A.C. Manalo and W. Karunasena, Mater. Des., 47, 424 (2013).

14. T.G. Rials, M.P. Wolcott and J.M. Nassar, J. Appl. Polym. Sci., 80, 546 (2001).

15. E. Sojstrom, Wood Chemistry Fundamentals and Applications, Academic Press, USA (1993).

16. D.P. Kamdem, H. Jiang, W. Cui, J. Freed and L.M. Matuana, Composites A, 35, 347 (2004).

17. J.-P. Kim, T.-H. Yoon, S.-P. Mun, J.-M. Rhee and J.-S. Lee, Bioresour. Technol., 97, 494 (2006).

18. G. Constantinescu and G. Cazacu, Rom. J. Physiol., 52, 375 (2007).

19. S. Panthapulakkal and M. Sain, Composites A, 38, 1445 (2007).

20. Y. Cui, S. Lee, B. Noruziaan, M. Cheung and J. Tao, Composites A, 39, 655 (2008).

21. A.K. Bledzki, A.A. Mamun and J. Volk, Composites A, 41, 480 (2010).

22. X. Guo, D. Xiang, G. Duan and P. Mou, Waste Manag., 30, 4 (2010).

23. S.Y. Leu, T.H. Yang, S.F. Lo and T.H. Yang, Construct. Build. Mater., 29, 120 (2012).

24. S.T. Sundar, A.G. McDonald and M. Wolcott, Proc. Progress in Wood Fibre-Plastic Composites Conference, May 10-11. Toronto, Ontario, Canada (2004).

25. R.M. Rowell and W.B. Banks, Water Repellency and Dimensional Stability of Wood, USDA Forest Service General Technical Report, FPL 50, FPL Madison Wisconsin, USA (1985).

26. R.G. Raj and B.V. Kokta, Proc. 49th Annual Technical Conference, Society of Plastic Engineers, Brookfield, CT, Montreal, Canada, pp. 1883-1885 (1991).

27. H. Gao, Y. Xie, R. Ou and Q. Wang, Composites A, 43, 150 (2012).

28. C. Li, Y. Zhang and Y. Zhang, Polym. Test., 22, 191 (2003).

29. L. Yang, F. Zhang, T. Endo and T. Hirotsu, Macromolecules, 36, 4709 (2003).

30. M. Sclavons, P. Franquinet, V. Carlier, G. Verfaillie, I. Fallais, R. Legras, M. Laurent and F.C. Thyrion, Polymer, 41, 1989 (2000). 